\title{
The role of transposable elements in the evolution of non-mammalian vertebrates and invertebrates
}

\author{
Noa Sela1,2, Eddo Kim and Gil Ast*1
}

\begin{abstract}
Background: Transposable elements (TEs) have played an important role in the diversification and enrichment of mammalian transcriptomes through various mechanisms such as exonization and intronization (the birth of new exons/introns from previously intronic/exonic sequences, respectively), and insertion into first and last exons. However, no extensive analysis has compared the effects of TEs on the transcriptomes of mammals, non-mammalian vertebrates and invertebrates.
\end{abstract}

Results: We analyzed the influence of TEs on the transcriptomes of five species, three invertebrates and two nonmammalian vertebrates. Compared to previously analyzed mammals, there were lower levels of TE introduction into introns, significantly lower numbers of exonizations originating from TEs and a lower percentage of TE insertion within the first and last exons. Although the transcriptomes of vertebrates exhibit significant levels of exonization of TEs, only anecdotal cases were found in invertebrates. In vertebrates, as in mammals, the exonized TEs are mostly alternatively spliced, indicating that selective pressure maintains the original mRNA product generated from such genes.

Conclusions: Exonization of TEs is widespread in mammals, less so in non-mammalian vertebrates, and very low in invertebrates. We assume that the exonization process depends on the length of introns. Vertebrates, unlike invertebrates, are characterized by long introns and short internal exons. Our results suggest that there is a direct link between the length of introns and exonization of TEs and that this process became more prevalent following the appearance of mammals.

\section{Background}

Transposable elements (TEs) are mobile genetic sequences that comprise a large fraction of mammalian genomes: $45 \%, 37 \%$ and $55 \%$ of the human, mouse and opossum genomes are made up of these elements, respectively [1-6]. TEs are distinguished by their mode of propagation. Short interspersed repeat elements (SINEs), long interspersed repeat elements (LINEs) and retrovirus-like elements with long-terminal repeats (LTRs) are propagated by reverse transcription of an RNA intermediate. In contrast, DNA transposons move through a direct 'cut-and-paste' mechanism [7]. TEs are not just 'junk' DNA but rather are important players in mammalian evolution and speciation through mechanisms such as exonization and intronization [8-11]. Alternative splicing of exonized TEs can be tissue specific $[12,13]$ and

* Correspondence: gilast@post.tau.ac.il

1 Department of Human Molecular Genetics, Sackler Faculty of Medicine, Tel Aviv University, Tel Aviv 69978, Israel

Full list of author information is available at the end of the article exonization contributes to the diversification of genes after duplication [14].

Most exonized TEs are alternatively spliced, which allows the enhancement of transciptomic and proteomic diversity while maintaining the original mRNA product [9-11,15,16]. Exonization can take place following insertion of a TE into an intron. However, most invertebrate introns are relatively short [17] and are under selection to remain as such due to the intron definition mechanism by which they are recognized [18-21]. Thus, there is presumably a selection against TE insertion into such introns. However, with the presumed transition from intron to exon definition during evolution [20,22], introns were freed from length constraints. This reduced the selection against insertion of TEs into introns and a large fraction of mammalian introns contain TEs, although only a small fraction are exonized [16]. For the most part, TEs have not been inserted within internal coding exons; they are found in first and last exons and in untranslated 
regions (UTRs), apparently the outcome of coding constraints [16].

The impact of TEs on the genomes of human [811,16,23-26], dog [4,5], cow [3], mouse [16] and opossum $[6,27]$ has been extensively studied. Bejerano and colleagues [28] have shown that SINEs that were active in non-mammalian vertebrates during the Silurian period are the source of ultra-conserved elements within mammalian genomes. However, with this exception there have been no systematic large-scale analyses of the impact of TEs on the transcriptomes of non-mammalian genomes. To address this issue we compiled a dataset of all TE families in the genomes of chicken (Gallus gallus), zebrafish (Danio rerio), sea squirt (C. intestinalis), fruit fly (Drosophila melanogaster) and nematode (Caenorhabditis elegans). We examined the location of each TE with respect to annotated genes. We found that the percentage of TEs within transcribed regions of these non-mammalian vertebrates and invertebrates is much lower than the percentage observed within mammals. We also found evidence for TE exonization in all species we examined. However, the magnitude of this process differed among the tested organisms; we detected a substantially higher level of exonizations in vertebrates (G. gallus and D. rerio) compared to invertebrates (D. melanogaster and C. elegans). There is a higher abundance of TEs in intronic sequences, and introns are much larger in vertebrates than in invertebrates, suggesting that TEs located in long introns provide fertile ground for testing new exons via the exonization process. Overall, the results we present suggest that TE exonization is a mechanism for transcriptome enrichment not only in mammals, but also in nonmammalian vertebrates as well as in invertebrates, albeit to a lesser extent.

\section{Results}

\section{Genome-wide analysis of TE insertions within the} transcriptomes of five non-mammalian species

To evaluate the effect of TEs on the transcriptomes of non-mammals, we analyzed the genomes of five nonmammalian vertebrates and invertebrates: G. gallus, D. rerio, C. intestinalis, D. melanogaster and C. elegans. To calculate the total number of TEs in each genome, the number of TEs in introns, and the number of TEs present within mRNA molecules, we downloaded EST and cDNA alignments and repetitive element annotations for these five genomes from the University of California Santa Cruz (UCSC) genome browser [24] (see Materials and methods and also [29]). Tables 1, 2, 3, 4 and 5 summarize our analyses for each of these species.

TEs have altered the transcriptomes of mammals and the examined non-mammalian genomes differently. First, the portion of the genome covered by TEs differs dramatically. In mammalian genomes, TEs occupy between 37\% and $52 \%$ of the genome $[1-6,30]$. In the five evaluated non-mammalian genomes, TEs account for approximately $10 \%$ of the genome sequence, with the exception of D. rerio, where TEs occupy $26.5 \%$ (Figure 1 ). The second important difference is related to the types of TEs observed. In mouse and human, SINEs are the most abundant TEs. In the G. gallus genome, LINEs (belonging to the family of CR1 repeats) account for $79 \%$ of all TEs. In the $D$. rerio genome, more than $75 \%$ of TEs are DNA transposons; whereas in D. melanogaster, LTRs are the most abundant TEs, accounting for $44 \%$ of the elements observed. Finally, DNA transposons account for $95 \%$ of TEs in C. elegans. These differences have influenced the transcriptomes of non-mammals: in contrast to SINEs, which are non-autonomous mobile elements that do not encode for proteins, all other families of TEs are autonomous and contain at least one open reading frame.

\section{Insertion of TEs within intronic sequences}

Deeper analysis of the non-mammalian genomes revealed that TEs are less likely to be fixed within transcribed regions relative to orthologous regions in human and mouse [16]. In G. gallus, D. rerio and C. intestinalis, $33.2 \%, 47.3 \%$ and $39.4 \%$ of TEs reside within introns, respectively, whereas in the human genome, approximately $60 \%$ of TEs reside within introns [16] $\left(x^{2}, P\right.$-value $=0$, for a comparison of TEs either in G. gallus, D. rerio, or $C$. intestinalis versus human). In the genome of D. melanogaster, the fraction of intronic TEs is $60 \%$, similar to that of mammals $\left(X^{2}, P\right.$-value $=0.3$ compared with human); in C. elegans 53\% of TEs reside within intronic sequences, significantly lower compared to human $\left(\chi^{2}, P\right.$ value $=1.1 \mathrm{e}-42)$. Among all TEs, LTRs have the lowest insertion levels within intronic sequences compared to other TE families in all genomes analyzed (Tables 1, 2, 3, 4 , and 5), as was also observed for human and mouse [16]. The lower level of invasion of TEs within intronic sequences in $D$. melanogaster may be due in part to the fact that a large fraction of TEs in Drosphila are LTR sequences that have a lower tendency than other TE families to reside within introns $[16,31]$.

We next evaluated the TE distribution and determined the length of introns that contain TEs (Figure 2). We analyzed all intronic sequences of human (total of 184,145 introns), mouse (total of 177,766 introns), G. gallus (total of 167,626 introns), D. rerio (total of 194,221 introns), C. intestinalis (total of 34,328 introns), D. melanogaster (total of 41,145 introns) and C. elegans (total of 98,695 introns) for TE insertions to determine the percentage of TE-containing introns (Figure 2a). The fraction of the introns that contain TEs in the non-mammalian vertebrates G. gallus and D. rerio is $21.3 \%$ and $44.3 \%$, respectively, substantially lower than that of mammals $(63.4 \%$ and $60.2 \%$ in human and mouse, respectively). The frac- 
Table 1: Transposable elements in Gallus gallus

\begin{tabular}{lrrrrrr}
\hline TE & Total & Intronic & $\begin{array}{r}\text { TEs in introns } \\
\text { within RefSeq }\end{array}$ & $\begin{array}{r}\text { TEs in introns of } \\
\text { non-RefSeq }\end{array}$ & $\begin{array}{r}\text { Exons within RefSeq } \\
\text { alignments* }\end{array}$ & $\begin{array}{r}\text { Exons in non-RefSeq } \\
\text { alignmentst }\end{array}$ \\
\hline SINE & 27 & $10(37 \%)$ & 1 & 9 & 0 & 0 \\
LINE & 188,302 & $65,035(34.5 \%)$ & 14,482 & 50,553 & 8 & 0 \\
LTR & 28,719 & $7,553(26.3 \%)$ & 1,501 & 6,052 & 1 & 8 \\
DNA & 20,808 & $6,554(31.4 \%)$ & 1,446 & 5,108 & 9 \\
\hline Total & 237,856 & $79,152(33.2 \%)$ & 17,430 & 61,722 & 61 \\
\hline
\end{tabular}

*Number of exons found within annotated RefSeq genes. ${ }^{\dagger}$ Number of exons for which ESTs are not found within annotated RefSeq genes.

tion of introns containing TEs in the deuterostome $C$. intestinalis is $33.4 \%$, very similar to the percentage in non-mammalian vertebrates. In contrast, the fraction of introns that contain TEs in invertebrates $D$. melanogaster and C. elegans is $1.7 \%$ and $5.6 \%$, respectively. These results indicate that only a very small portion of introns in invertebrates contain TEs ( 2 to 5\%) compared to 20 to $40 \%$ of introns in non-mammalian vertebrates and approximately $60 \%$ in mammals.

We also examined the average length of introns containing TEs. In C. elegans the median length of an intron containing a TE is approximately $700 \mathrm{bp}$ (after subtracting TE length, the median intron size is $477 \mathrm{bp}$ ), compared to approximately 3,000 bp in human, mouse, chicken and zebrafish. The median length of introns that contain TEs in the fruit fly is around 6,000 bp (after subtracting the TE length, the median intron length is 5,822 $\mathrm{bp})$, whereas the median length of introns in fruit fly is only 72 bp [17] (Figure 2b, c). Therefore, the introns in fruit fly that contain TEs are presumably under different selective pressure than the vast majority of introns in this organism; we assume that these TE-containing introns are not selected via the intron definition mechanism [19]. In general, we found a positive correlation between the fraction of introns containing TEs and median length of introns (Figure 2c), implying that TE insertions have played a role in the evolution of intron size.

Previous analysis of human and mouse transcriptomes revealed that there is a biased insertion and fixation of some families of TEs within intronic sequences [16]: L1 and LTRs are most often fixed in their antisense orientation relative to the mRNA molecule. Our current analysis also revealed a bias toward antisense fixations of LTR sequences within G. gallus, D. rerio and D. melanogaster genomes (Additional file 1). This biased insertion is also correlated with a lower tendency of LTRs to reside within intronic sequences relative to other families of TEs (see Tables 1, 2, 3, 4 and 5 for data on non-mammalian genomes and [16] for data on human and mouse). A bias toward antisense orientation was also observed for DNA transposons in G. gallus and D. melanogaster and for LINEs in D. melanogaster. These biased insertions are presumably due to potential for co-transcription of TEs that already contain coding sequences. Insertion in a sense orientation would introduce another promoter into the transcribed region, which is likely to be deleterious and therefore selected against.

\section{Exonizations within vertebrates and invertebrates}

In mammals, new exonizations resulting from TEs are mostly alternatively spliced cassette exons

Table 2: Transposable elements in Danio rerio

\begin{tabular}{|c|c|c|c|c|c|c|}
\hline TE & Total & Intronic & $\begin{array}{l}\text { TEs in introns } \\
\text { within RefSeq }\end{array}$ & $\begin{array}{r}\text { TEs in introns of } \\
\text { non-RefSeq }\end{array}$ & $\begin{array}{r}\text { Exons within RefSeq } \\
\text { alignments* }\end{array}$ & $\begin{array}{r}\text { Exons in non-RefSeq } \\
\text { alignments } t\end{array}$ \\
\hline SINE & 259,684 & $113,926(43.9 \%)$ & 46,679 & 67,247 & 14 & 121 \\
\hline LINE & 80,412 & $37,228(46.3 \%)$ & 14,671 & 22,557 & 2 & 4 \\
\hline LTR & 53,028 & $21,496(40.5 \%)$ & 6,761 & 14,735 & 2 & 1 \\
\hline DNA & $1,208,155$ & $585,408(48.4 \%)$ & 257,438 & 327,970 & 37 & 72 \\
\hline Total & $1,601,279$ & $758,058(47.3 \%)$ & 325,549 & 432,509 & 55 & 198 \\
\hline
\end{tabular}

*Number of exons for which their ESTs are found within annotated RefSeq genes.

${ }^{\dagger}$ Number of exons for which their ESTs are not found within annotated RefSeq genes. 
Table 3: Transposable elements in Ciona intestinalis

\begin{tabular}{|c|c|c|c|c|c|c|}
\hline TE & Total & Intronic & $\begin{array}{l}\text { TEs in introns } \\
\text { within RefSeq }\end{array}$ & $\begin{array}{r}\text { TEs in introns of } \\
\text { non-RefSeq }\end{array}$ & $\begin{array}{r}\text { Exons within } \\
\text { RefSeq } \\
\text { alignments* }\end{array}$ & $\begin{array}{r}\text { Exons in non-RefSeq } \\
\text { alignments }\end{array}$ \\
\hline SINE & 51,021 & 20,360 (39.9\%) & 826 & 19,534 & 0 & 3 \\
\hline LINE & 29,369 & $11,172(38 \%)$ & 493 & 10,679 & 0 & 0 \\
\hline LTR & 491 & $112(22.8 \%)$ & 2 & 110 & 0 & 0 \\
\hline DNA & 55,300 & $22,056(39.9 \%)$ & 1,025 & 21,031 & 0 & 9 \\
\hline Total & 136,181 & 53,700 (39.4\%) & 1,851 & 51,849 & 0 & 12 \\
\hline
\end{tabular}

*Number of exons for which their ESTs are found within annotated RefSeq genes.

${ }^{\dagger}$ Number of exons for which their ESTs are not found within annotated RefSeq genes.

$[10,11,15,16,26,32,33]$. In non-mammalian genomes, the level of alternative splicing is lower than that of mammals, with the exception of chicken, where levels of alternative splicing are comparable to those in human [34]. We analyzed the splicing patterns of the TE-derived exons in the four non-mammalian species that contain TE-derived exons; the analysis was based on alignment data between EST/cDNA sequences and their corresponding genomic regions. The TE-derived exons in $D$. rerio, C. intestinalis and C. elegans were predominantly alternatively spliced (Figure 3 ), a phenomenon similar to that found in mammals, suggesting that similar evolutionary constraints (reviewed in $[22,26,35]$ ) affect exonizations of mammals and species outside the mammalian class. In D. melanogaster, there are no exonized TEs in which one of the splice sites results from the TE sequence. G. gallus is an exception: in this species many TE exonizations were constitutively spliced. However, this observation may be a result of a substantially lower number of ESTs available for G. gallus (Additional file 2). Without sufficient EST data, identification of alternatively spliced exons is difficult and exons may be mistakenly classified as constitutively spliced. We will need to re-evaluate this statement once additional EST coverage becomes available for G. gallus.

Most TE exonizations occur in genomic loci that are not annotated as genes by the RefSeq [36,37] or Ensembl $[38,39]$ databases. It may be that these genes are speciesspecific and are not annotated due to a lack of homologs; alternatively, these may be non-protein coding genes. Of the exonizations found in annotated genes, 66 to $87 \%$ are found within the coding sequence (Additional file 3). Exonizations in non-mammals frequently disrupted the open reading frame of a protein, similar to results previously reported for human and mouse. In G. gallus, D. rerio and C. intestinalis only 38 to $50 \%$ of the exonized TEs have lengths divisible by three and therefore maintain the original coding sequence (Additional file 3 ).

In D. melanogaster, we found no evidence for exonizations using current ESTs or cDNA. We did identify three cases in which TEs were inserted into internal exons, all within the coding sequence (see Figure 4 and Additional file 4 for exon sequences). In these cases, the length of the inserted TEs (LINEs) was found to be divisible by three and the sequences did not contain stop codons. Thus, the insertion of these TEs into the coding exons did not alter the reading frame of the downstream exons, but rather

Table 4: Transposable elements in Drosophila melanogaster

\begin{tabular}{lrrrrrr}
\hline TE & Total & Intronic & $\begin{array}{c}\text { TEs in introns } \\
\text { within RefSeq }\end{array}$ & $\begin{array}{r}\text { TEs in introns of } \\
\text { non-RefSeq }\end{array}$ & $\begin{array}{r}\text { Exons within RefSeq } \\
\text { alignments* }\end{array}$ & $\begin{array}{c}\text { Exons in non-RefSeq } \\
\text { alignmentst }\end{array}$ \\
\hline SINE & 0 & 0 & 0 & 0 & 0 & 0 \\
LINE & 4,755 & $2,964(62 \%)$ & 1,258 & 1,706 & 0 & 0 \\
LTR & 10,259 & $5,394(52 \%)$ & 2,014 & 3,380 & 0 & 0 \\
DNA & 8,028 & $5,560(69 \%)$ & 3,231 & 2,329 & 0 \\
\hline Total & 23,042 & $13,918(60 \%)$ & 6,503 & 7,415 & 0 \\
\hline
\end{tabular}

*Number of exons for which their ESTs are found within annotated RefSeq genes.

${ }^{\dagger}$ Number of exons for which their ESTs are not found within annotated RefSeq genes. 
Table 5: Transposable elements in Caenorhabditis elegans

\begin{tabular}{lrrrrrr}
\hline TE & Total & Intronic & $\begin{array}{r}\text { TEs in introns } \\
\text { within RefSeq }\end{array}$ & $\begin{array}{r}\text { TEs in introns of } \\
\text { non-RefSeq }\end{array}$ & $\begin{array}{r}\text { Exons within RefSeq } \\
\text { alignments* }\end{array}$ & $\begin{array}{r}\text { Exons in non-RefSeq } \\
\text { alignmentst }\end{array}$ \\
\hline SINE & 524 & $243(46 \%)$ & 230 & 13 & 0 & 0 \\
LINE & 428 & $103(24 \%)$ & 90 & 13 & 0 & 0 \\
LTR & 606 & $137(22 \%)$ & 126 & 549 & 4 & 0 \\
DNA & 32,977 & $17,724(53 \%)$ & 17,175 & 586 & 4 \\
\hline Total & 34,535 & $18,207(53 \%)$ & 17,621 & 5 & 0 \\
\hline
\end{tabular}

*Number of exons for which their ESTs are found within annotated RefSeq genes.

${ }^{\dagger}$ Number of exons for which their ESTs are not found within annotated RefSeq genes.

added new amino acid sequence to the proteins. These insertions result in extremely long exons $(668,2,025$ and $4,077 \mathrm{bp})$. One of these exons is flanked by very short introns (82 and 68 bp for the upstream and downstream introns; Figure 4c) and two are flanked by a short downstream intron and a long upstream intron $(85$ and $70 \mathrm{bp}$ for the downstream introns and 1,003 and $689 \mathrm{bp}$ for the upstream introns; Figure 4a, b). In mammals, no evidence was found for TE insertions into coding exons $[15,16]$. We assume that this difference between mammals and Drosophila is due to the fact that in D. melanogaster the intron definition mechanism is dominant, which allows the lengthening of exons in a short-intron environment [19].

We have recently shown evidence for transduplication of protein coding genes within DNA transposons in $C$. elegans [40]. In this analysis, we found that DNA transposons have also influenced the coding sequence of C. elegans genes by means of exonization. One such example is an alternatively spliced exon of $73 \mathrm{bp}$ in the coding sequence of a hypothetical protein (Y71G12A.2). The

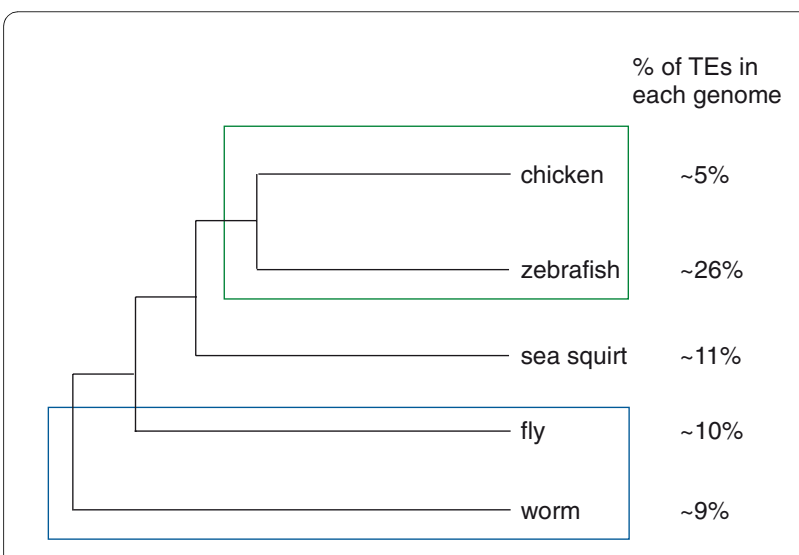

Figure 1 Non-mammalian vertebrate and invertebrate genomes have lower levels of TEs than mammalian genomes. Evolutionary trees for chicken [30], zebrafish, sea squirt [62], Drosophila [63] and worm [63]. Percentages of TEs in each genome are shown on the right. accession number of the RefSeq sequence that contains the exonization is [NM_058514]; the accession number of the RefSeq sequence without the exonization is [NM_001129082] (both RefSeq mRNA sequences have been reviewed). The gene is conserved within nematodes (C. remanei, C. briggsae, C. brenneri and C. japonica). It should be noted that only a single $C$. elegans individual has been sequenced and this event might be restricted to this individual. However, this event does suggest that an exonization mechanism operates in nematodes.

New exonizations resulting from TEs were found in the non-vertebrate deuterostome $C$. intestinalis (9 exonizations; Table 3) and in much larger quantities in vertebrates (70 in G. gallus and 253 in D. Rerio; Tables 1 and 2, respectively). The number of exonizations was not directly correlated to the number of ESTs available for each genome, suggesting that our results reflect a true difference in the extent of exonization across organisms. There are 599,785 ESTs for G. gallus, 1,380,071 ESTs for D. rerio, 1,205,674 ESTs for C. intestinalis, 573,981 ESTs for D. melanogaster and 352,044 ESTs for C. elegans (Additional file 5). Most exonizations found in G. gallus result from the CR1 LINE element, which is the most abundant TE within the G. gallus genome.

In the zebrafish genome, like that of mammals, the most abundant TEs are SINEs. About 68\% (77,436 copies) of zebrafish TEs are intronic SINEs that belong to the HE1 family of SINEs; these HE1 SINEs comprise almost $10 \%$ of the zebrafish genome [41]. The HE1 are tRNAderived SINEs with a 402-bp consensus sequence are also found in elasmobranches (the subclass of cartilaginous fish) [42]. The HE1 family is the oldest known family of SINEs, dated to 200 million years ago [42]. The HE1 SINEs were previously shown to be the source of mutational activity in the zebrafish genome and have been used as a tool for characterization of zebrafish populations [41]. SINEs have resulted in a substantial number of new exons (135 exons; Table 2) and 84.4\% (114 exons) are derived from HE1 SINEs. Of the 114 cases of exoniza- 


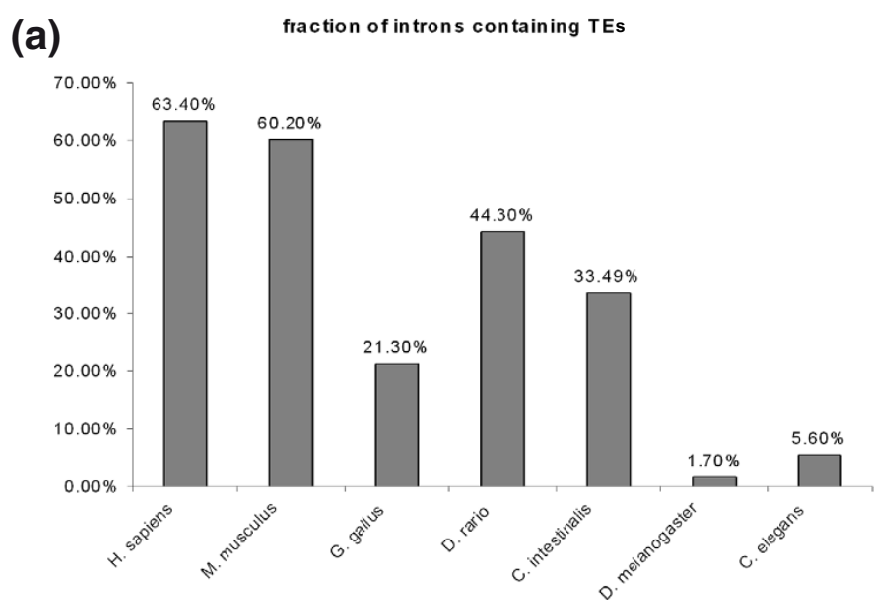

(b)
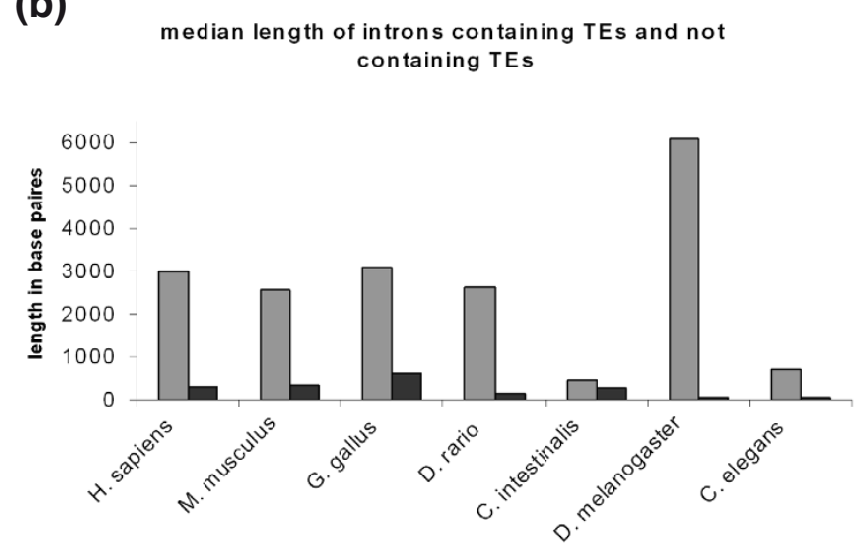

(c)

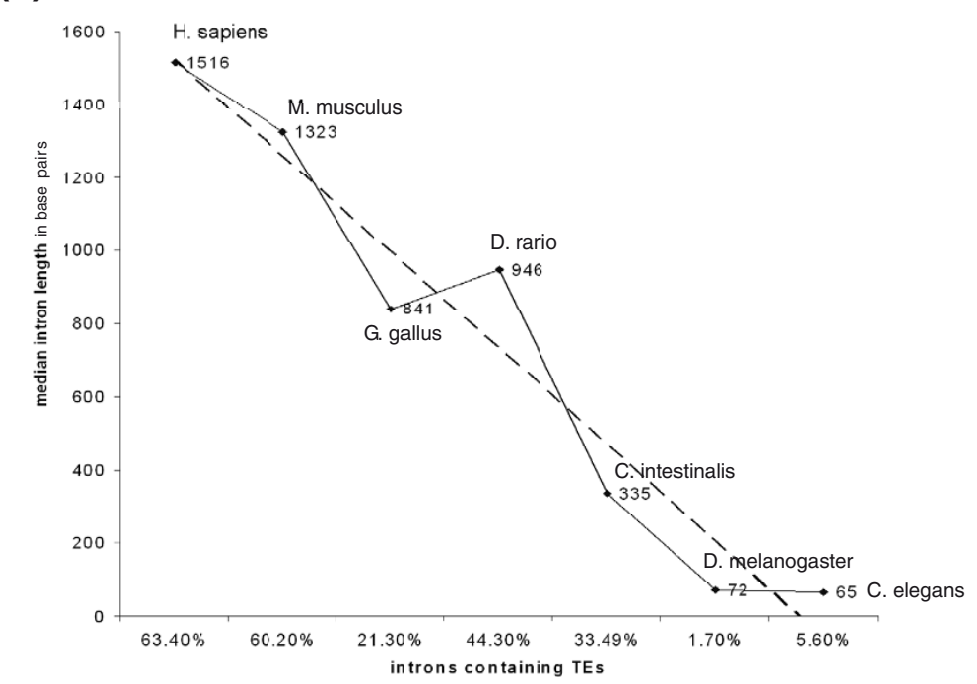

Figure 2 The fraction of introns containing TEs and their median lengths in non-mammalian and mammalian transcriptomes. (a) The fraction of TE-containing introns within five non-mammalian genomes compared to that of human (Homo sapiens) and mouse (Mus musculus) (for details see Materials and methods). (b) A graph of the median length of introns containing TEs compared to that of introns without TEs (marked in grey and black, respectively) in the different organisms. (c) Positive correlation between median intron length and the fraction of TEs containing introns. Intron lengths were taken from [17]. 


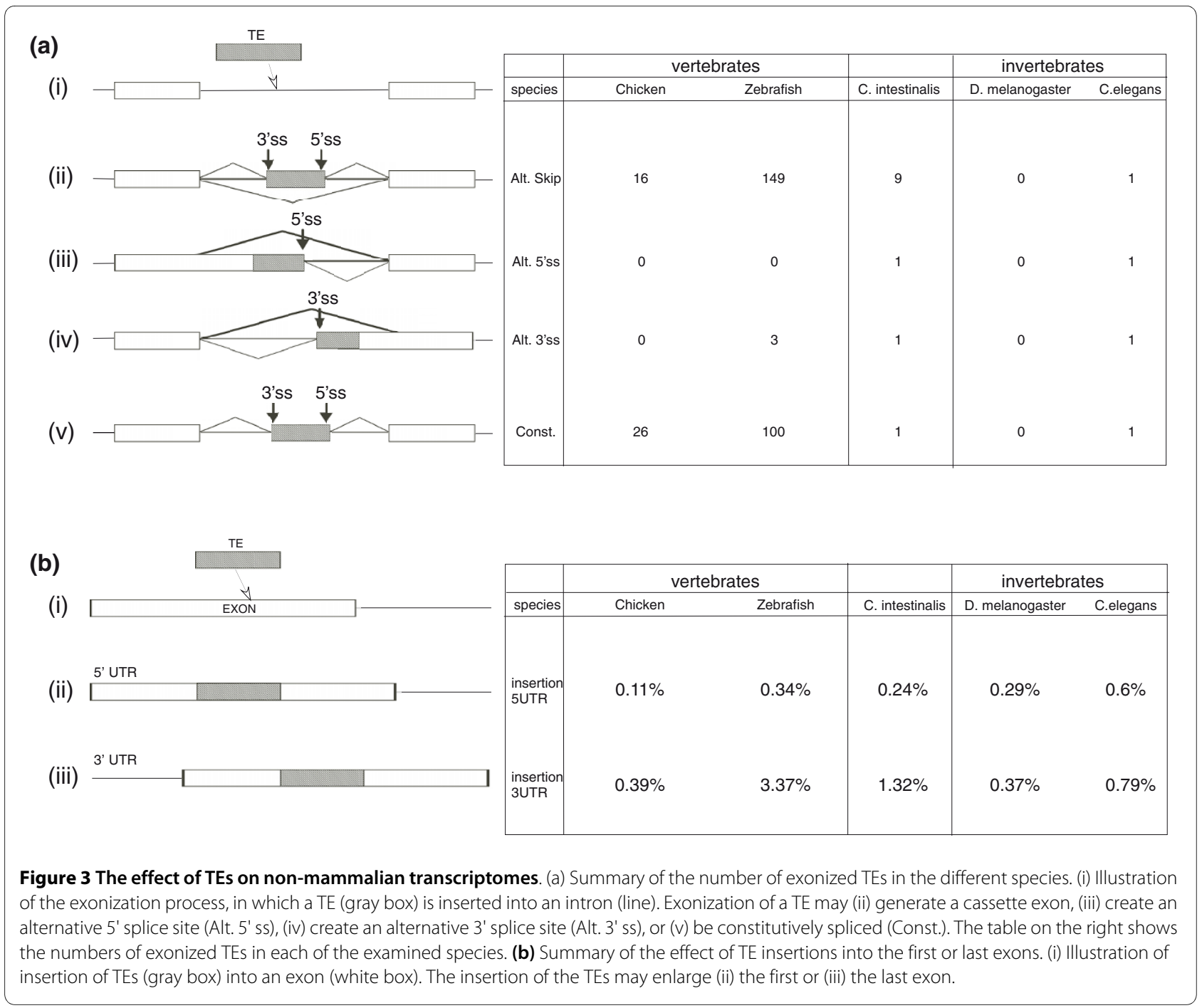

tions from HE1 elements, 69 insertions were in the sense orientation and 45 in the antisense orientation with respect to the coding sequence. These results suggest that there is no statistical preference for exonization in a specific orientation $\left(X^{2}, P\right.$-value $\left.=0.14\right)$. A typical SINE contains a poly(A) tail. Most exonizations originated from SINEs (Alu, B1, mammalian interspersed repeat (MIR)) are from elements inserted into introns in the antisense orientation relative to the coding sequence $[10,15,16]$. When SINEs with poly(A) insert into introns in the antisense orientation the poly(A) tail becomes a poly(U) in the mRNA precursor and thus can serve as a polypyrimidine tract for mRNA splicing [9]. The lack of a preference for exonization in a specific orientation of HE1 in zebrafish is presumably because of the absence of a poly(A) tail from the sequence of this SINE [43]. The tRNA-related, 5'-conserved regions of the HE1 element contain sequences that serve as 3 ' and 5 ' splice sites (Figure $5 \mathrm{a})$. When a sense HE1 region is exonized, the exonization is within the $5^{\prime}$ conserved area, whereas exonizations from HE1 elements in the antisense orientation encompass the entire HE1 sequence (Figure 5). Finally, DNA repeat elements are also substantial contributors of new exons in zebrafish (109 exons; Table 2). The exonization of DNA repeats is not biased to one of the orientations $\left(\chi^{2}, P\right.$-value $\left.=0.13\right)$.

\section{TE insertions into the first and last exons}

Our analysis shows that the influence of TEs on the transcriptomes of non-mammals is not limited to the creation of new internal exons: TEs also modified the mRNA by insertion into the first or last exon of a gene. This type of insertion causes an elongation of the first or last exons and usually affects the UTR (Figure 4b). In human, this type of insertion has been shown to create new non-conserved polyadenylation signals [44], influence the level of gene expression [45] and create new microRNA targets $[46,47]$. 
(a) cno gene

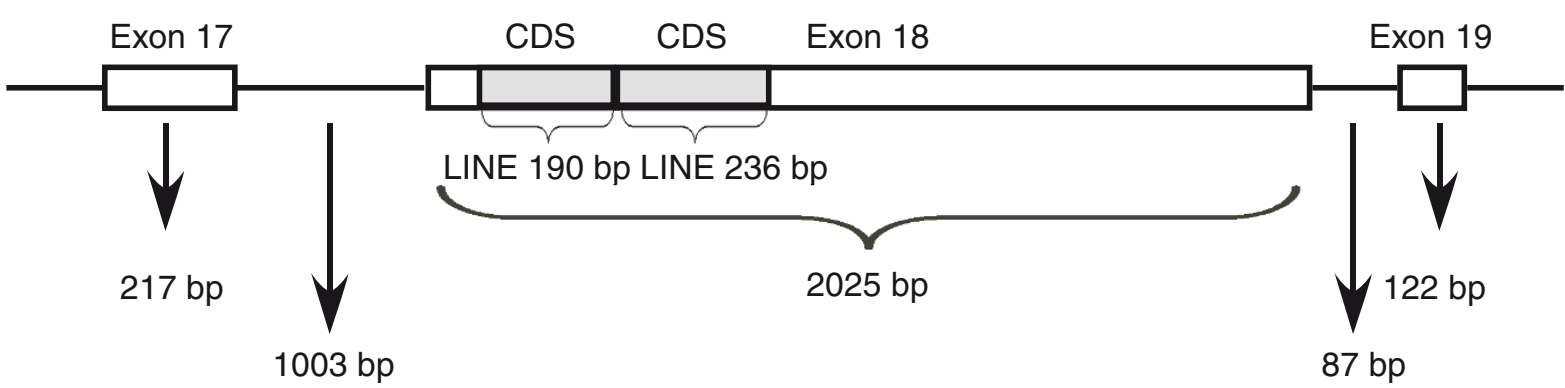

(b) CG14821 gene

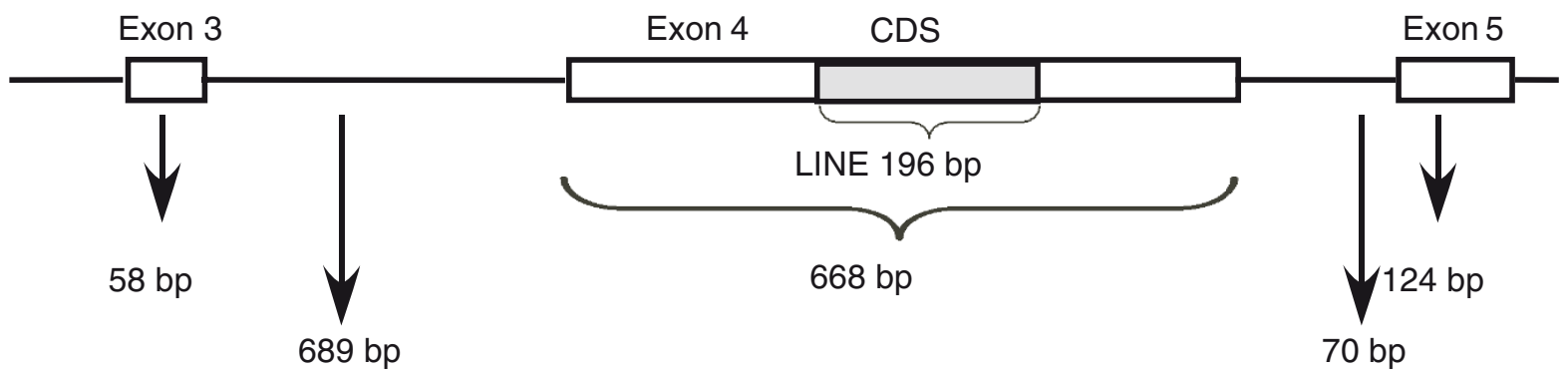

(c) nej gene

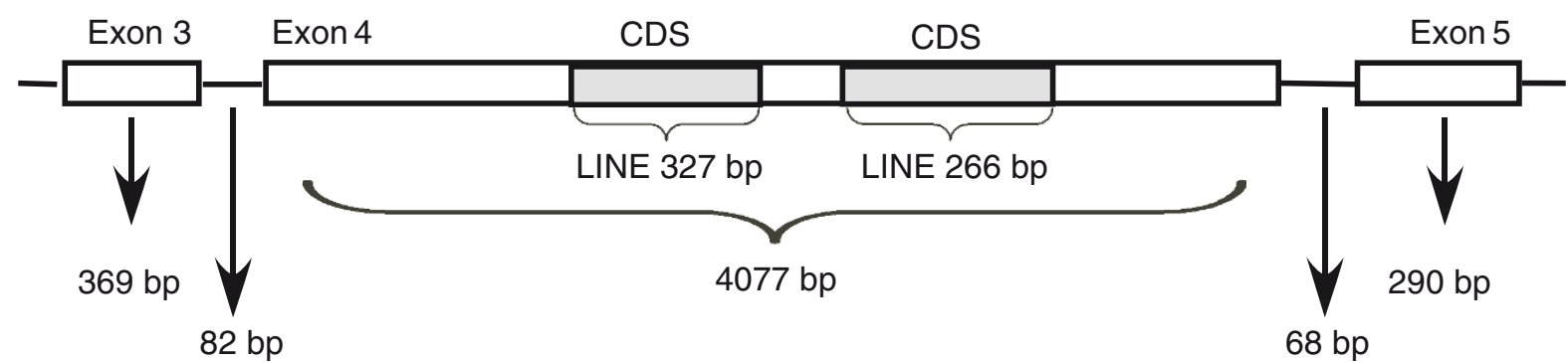

Figure 4 Three cases of TE insertions into internal exons in D. melanogaster. Schematic representations of TE insertions into Drosophila internal exons. White boxes and lines represent exons and introns, respectively. The grey boxes show insertion of TEs into exons. The TE family is indicated beneath the gray box, along with the length of each inserted TE. Lengths of the introns and exons flanking the inserted exon are indicated. Genes with insertions are (a) cno, (b) CG14821 and (c) nej. CDS, coding sequence.

For the analysis of the number of TE insertions within the first or last exons in chicken, zebrafish, fruit fly and nematode, we used the UCSC annotated RefSeq genes and examined those full-length sequences in which the entire transcript is annotated and a consensus mRNA sequence exists. Our results indicate that TEs occupy a lower percentage of the base pairs within the first and last exons in mouse, chicken, zebrafish, C. intestinalis, D. melanogaster and C. elegans than do TEs in the first and last exons of human (Additional files 5 and 6). Our previous analysis showed that in human annotated genes, the aver- age lengths of the first and last exons are 465 and 1,300 bp, respectively, and in mouse genes the first exon has an average length of $393 \mathrm{bp}$ and the last exon an average length of 1,189 bp [16]. The average lengths of the first and last exons in the non-mammalian species are shown in Figure 6 (see also Additional files 5 and 6); all have average exon lengths shorter than those of human and mouse. The fly has, on average, the longest first exons among the non-mammalian species, whereas the chicken genome contains the longest last exons on average (Figure 6). 
(a) HEl bullhead shark
HEl Danio Rario conservation

HE 1 bull head shark HEl Danio Rario conservation

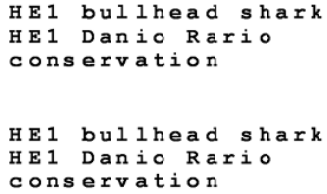

(b)

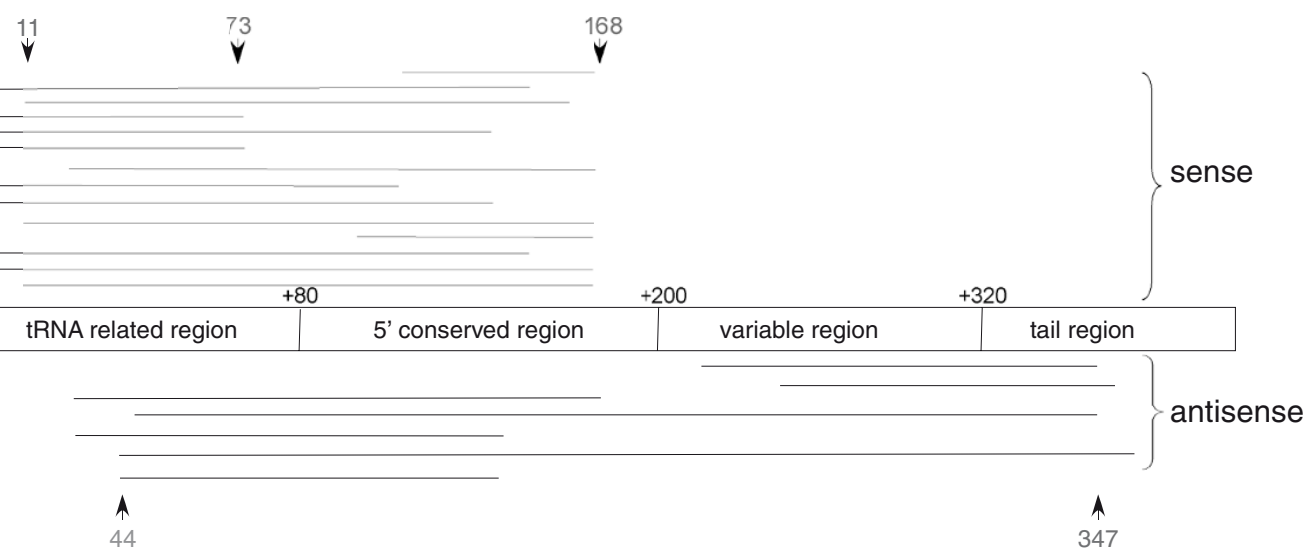

Figure 5 HE-1 SINE exonization in zebrafish. (a) Alignment of the HE1 SINE from D. rerio and the HE1 SINE from bullhead shark showing the different sections within the transposable element according to [43]. The letters y and $r$ denote pyrimidine and purine, respectively. (b) Non-redundant distribution and orientation of exonized HE1 SINE sequences in which both the 5' and 3' splice sites are within the HE1 SINE sequence. The exonized HE1 SINE sequence regions are aligned against an HE1 SINE consensus element. Each line is a different EST showing exonizations and the box in the middle represents the HE1 element. The number of cases that select that site as a 5 ' splice site $(73,168,44)$ or as a 3 ' splice site $(11,347)$ are shown. Exonizations in the sense and antisense orientations are shown above and below the schematic representation of the HE1. 


\section{Discussion}

In this study, we examined the influence of TEs on the transcriptomes of five species, including two vertebrates, one non-vertebrate deuterostome and two invertebrates. We compared our data to previous results generated for two mammalian species (human and mouse) [16]. We observed significant differences between vertebrates and invertebrates regarding the exonizations that have resulted from TE insertion. In chicken and zebrafish, we found dozens of exonizations: 70 exons were a result of TE insertions in G. gallus and 153 in D. rerio. Lower on the evolutionary tree, TEs were much less frequently exonized, if at all. In the deuterostome $C$. intestinalis, we found only 12 exons that resulted from TEs and none were observed in D. melanogaster and C. elegans.

The prevalence of exonizations within human and mouse (around 1,800 new exons in human and around 500 new exons in mouse [16]) is mainly attributed to the existence of very large introns and the dominance of the exon definition mechanism for splice site selection in mammals [48]. Invertebrates, in contrast, have short introns and long exons [17]. The transition from the intron definition mechanism used by invertebrates to that of exon definition during evolution presumably reduced selective pressure on intron length, which probably allowed insertion of TEs into intron sequences without deleterious consequences $[48,49]$. As could be expected due to the difference in the length of introns, the number of TEs located in intron sequences is substantially lower in the non-mammalian genomes compared to mammalian genomes. One might expect that in organisms where the splicing machinery functions via the intron definition mechanism, insertion of TEs into the longer coding exons would be prevalent. However, only three cases of such insertions were detected in the D. melanogaster genome, suggesting that this mechanism of transcriptome enrichment is evolutionarily unfavorable. It is likely that TE insertions into coding exons are not propagated as these events would alter the coding sequence immediately upon insertion. A previous genome-wide analysis of TEs in Drosophila and their association with gene location found a small number of fixed TEs [50]. However, other analyses have shown that TEs have played an important role in adaptation of fruit flies [51]. One of the most significant reports was that of the truncation of the CHKov1 gene by a TE leading to resistance to pesticides [52].

SINEs and LINEs were shown in many publications to be good substrates for the exonization process because of their special structure $[9,11,15,16,26]$. In mammalians and other vertebrates higher level of SINEs and LINEs within intron sequences gave rise to a greater level of exonization due to the pre-existence of splice site-like sequences, such as the polypyrimidine tract and putative 5 ' splice sites $[9,11,15,16,26]$.

TEs are often inserted into exonic regions that are part of UTRs. Our analysis indicated that, on average, the size of the last exons is longer in mammals compared to vertebrates and more so in invertebrates. The differences in the length of the last exons are correlated with an increase in the percentage of TEs inserted into last exons. Insertions of TEs into UTRs may alter levels of gene expression, create new targets for microRNA binding, or even result in precursors for new microRNAs $[46,47,53]$. Presumably, the increase in the size of the last exons and in the percentage of TEs within these exons from invertebrates to mammals may have led to the high level of regulatory complexity observed in higher organisms. Exonization of TEs is widespread in mammals, less so in non-mammalian vertebrates, and very low in invertebrates.

\section{Conclusions}

Our results suggest that there is a direct link between the length of introns and exonization of TEs and that this process became more prevalent following the appearance of mammals.

\section{Materials and methods}

\section{Dataset of TEs within coding regions of five species}

Chicken (galGal3, May 2006), zebrafish (danRer4, March 2006), fruit fly (dm2, April 2004), C. elegans (ce2, March 2004) and sea squirt (ci2, March 2005) genome assemblies were downloaded, along with their annotations, from the UCSC genome browser database [24,54]. EST and cDNA mappings were obtained from chrN_intronEST and chrN_mrna tables, respectively. TE mapping data were obtained from chrN_rmsk tables and $\mathrm{TE}$ sequences were retrieved from genomic sequences using the mapping data. A TE was considered intragenic if there was no overlap with ESTs or cDNA alignments; it was considered intronic if it was found within an alignment of an EST or cDNA defined as an intronic region. Finally, a TE was considered exonized if it was found within an exonic part of an EST or cDNA (except the first or last exon of the EST/cDNA), and possessed canonical splice sites. Next, we associated the intronic and exonized TEs with genomic positions of protein-coding genes by comparisons with RefSeq [55] gene tables from the UCSC table browser [54]. Positions of the TE hosting intron/ exon and the mature mRNA were calculated using the gene tables. Association of the gene with the mRNA and protein accessions and to descriptions from RefSeq and Swiss-Prot was done through the kgXref and refLink tables in the UCSC genome browser database [54]. All data used have been published [22,29]. 
(a)

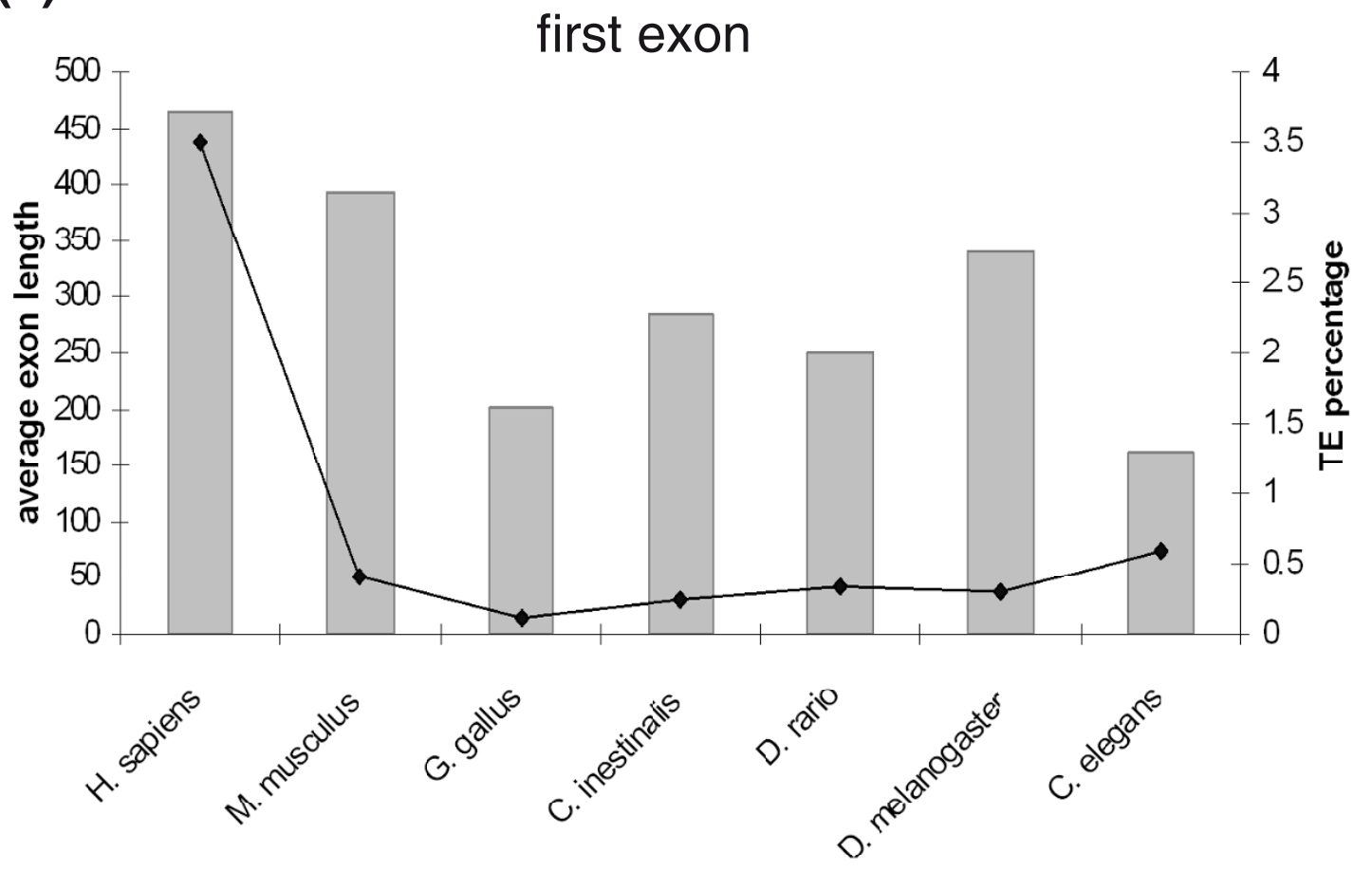

(b)

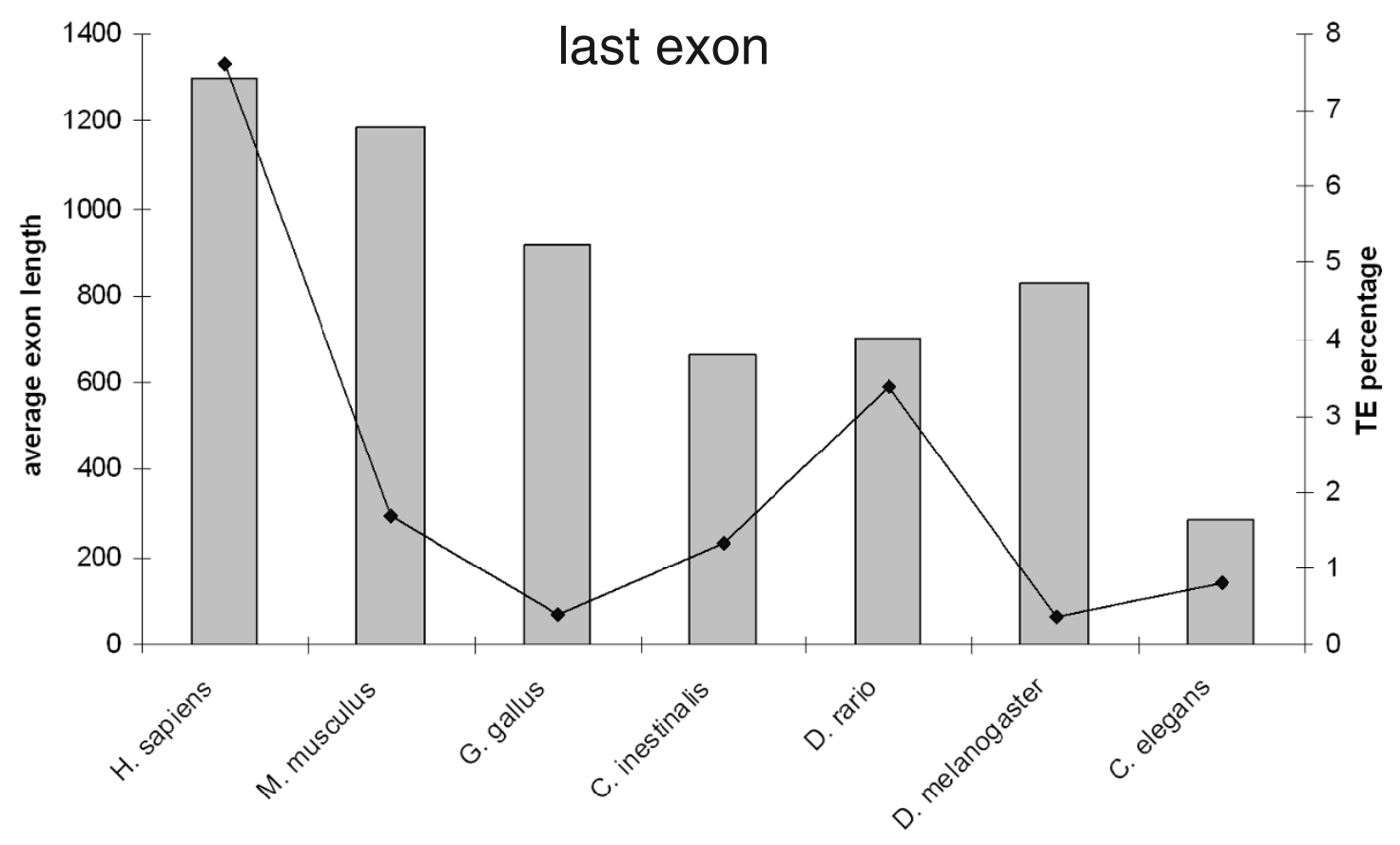

Figure 6 Average lengths of first and last exons compared to the fraction of TEs inserted into exons. (a) The $y$-axis indicates average length of first exon in the six examined organisms (bars) and the percentage of base pairs that originated from TEs (line). (b) Similar analysis for last exons. Note that the $y$-axes are different in scale. 


\section{Analysis of retroelement insertions within the first and last exons and assessment of UTR fraction in known genes}

The tables refGene and refLink were used to examine the relative lengths of the UTRs and the coding sequences within chicken, zebrafish, sea squirt, fruit fly and nematode genes and to find the first and last exons. The analysis of TE content was done using the RepeatMasker software [38] and repbase [56,57].

\section{Estimation of the fraction of TEs within introns}

We determined the TE fraction within intronic sequences using the UCSC genome browser and GALAXY [54,58,59]. Introns of chicken (G. gallus, build 1.1), zebrafish (D. rerio, release Zv4), C. elegans (release 2003) and D. melanogaster (build 4.1) were extracted from the Exon-Intron Database [60,61]. When alternatively spliced isoforms of the same gene were present, only the first annotated isoform was extracted; all other isoforms were excluded in order to avoid redundancy. The analysis of the TE content was done using RepeatMasker software and repbase [56,57]. In the case of $C$. intestinalis, the analysis of 34,328 intronic sequences was done using the GALAXY server [59] and UCSC genome browser tables [54].

\section{Statistical analysis}

For the comparative analysis of insertions within introns of various species we used a contingency table $x^{2}$ test. In cases where the contingency table was a $2 \times 2$ table, the Fisher's exact test was used. To assess the tendency of exonizations to occur within UTRs we used the goodness-of-fit $X^{2}$ test. The null hypothesis was the fraction of the UTR and coding sequence within the RefSeq gene list of chicken, zebrafish, sea squirt, fruit fly and C. elegans. The calculation of $P$-values for differences between two populations was measured according to the data distribution. The Kolmogorov-Smirnov test was used to test for normal distribution. The $t$-test was used to calculate statistical differences.

\section{Additional material}

\section{Additional file 1 Number of sense/antisense TE insertions within} intronic sequences

Additional file 2 Number of exonizations versus number of ESTs.

Additional file 3 Number of exonizations found in the coding

sequence or UTR (within annotated genes).

Additional file 4 Sequences of the internal $D$. melanogaster exons with TE insertions

Additional file $\mathbf{5}$ Average lengths and average TE percentages in first exons

Additional file 6 Average lengths and average TE percentages in last exons

\section{Abbreviations}

bp: base pair; EST: expressed sequence tag; LINE: Iong interspersed element LTR: long interspersed repeat; SINE: short interspersed element; TE: transposable element; UTR: untranslated region.

\section{Authors' contributions}

NS carried out the computational analysis. NS and GA conceived of the study. EK gave professional advice regarding interpretation of results. NS, EK and GA drafted the manuscript

\section{Acknowledgements}

The authors thank Wojciech Makalowski and Gyorgy Abrusan for stimulating discussions. This work was supported by the Cooperation Program in Cancer Research of the Deutsches Krebsforschungszentrum (DKFZ) and Israel's Minis try of Science and Technology (MOST) and by a grant from the Israel Science Foundation (40/05), ICRF, DIP and EURASNET. NS is supported by the LMU excellence fellowship.

\section{Author Details}

'Department of Human Molecular Genetics, Sackler Faculty of Medicine, Tel Aviv University, Tel Aviv 69978, Israel and 2Department of Biology I, LudwigMaximilians-University Munich (LMU) Großhaderner Str. 2, D-82152, Planegg Martinsried, Germany

Received: 17 March 2010 Revised: 27 April 2010

Accepted: 2 June 2010 Published: 2 June 2010

\section{References}

1. Lander ES, Linton LM, Birren B, Nusbaum C, Zody MC, Baldwin J, Devon K, Dewar K, Doyle M, FitzHugh W, Funke R, Gage D, Harris K, Heaford A Howland J, Kann L, Lehoczky J, LeVine R, McEwan P, McKernan K, Meldrim J, Mesirov JP, Miranda C, Morris W, Naylor J, Raymond C, Rosetti M, Santos $R$, Sheridan A, Sougnez C, et al.: Initial sequencing and analysis of the human genome. Nature 2001, 409:860-921.

2. Waterston RH, Lindblad-Toh K, Birney E, Rogers J, Abril JF, Agarwal P, Agarwala R, Ainscough R, Alexandersson M, An P, Antonarakis SE, Attwood J, Baertsch R, Bailey J, Barlow K, Beck S, Berry E, Birren B, Bloom T, Bork P, Botcherby M, Bray N, Brent MR, Brown DG, Brown SD, Bult C, Burton J, Butler J, Campbell RD, Carninci P, Cawley S, et al.: Initial sequencing and comparative analysis of the mouse genome. Nature 2002, 420:520-562.

3. Almeida LM, Silva IT, Silva WA Jr, Castro JP, Riggs PK, Carareto CM, Amaral ME: The contribution of transposable elements to Bos taurus gene structure. Gene 2007, 390:180-189.

4. Wang W, Kirkness EF: Short interspersed elements (SINEs) are a major source of canine genomic diversity. Genome Res 2005, 15:1798-1808.

5. Cordaux R, Batzer MA: Teaching an old dog new tricks: SINEs of canine genomic diversity. Proc Natl Acad Sci USA 2006, 103:1157-1158.

6. Gentles AJ, Wakefield MJ, Kohany O, Gu W, Batzer MA, Pollock DD, Jurka J: Evolutionary dynamics of transposable elements in the short-tailed opossum Monodelphis domestica. Genome Res 2007, 17:992-1004.

7. Hedges DJ, Batzer MA: From the margins of the genome: mobile elements shape primate evolution. Bioessays 2005, 27:785-794

8. Deininger PL, Batzer MA: Mammalian retroelements. Genome Res 2002 , 12:1455-1465

9. Lev-Maor G, Sorek R, Shomron N, Ast G: The birth of an alternatively spliced exon: 3' splice-site selection in Alu exons. Science 2003, 300:1288-1291.

10. Sorek R, Ast G, Graur D: Alu-containing exons are alternatively spliced. Genome Res 2002, 12:1060-1067.

11. Sorek R, Lev-Maor G, Reznik M, Dagan T, Belinky F, Graur D, Ast G: Minimal conditions for exonization of intronic sequences: 5 ' splice site formation in alu exons. Mol Cell 2004, 14:221-231.

12. Lin L, Shen S, Tye A, Cai JJ, Jiang P, Davidson BL, Xing Y: Diverse splicing patterns of exonized Alu elements in human tissues. PLoS Genet 2008 , 4:e1000225

13. Mersch B, Sela N, Ast G, Suhai S, Hotz-Wagenblatt A: SERpredict: detection of tissue- or tumor-specific isoforms generated through exonization of transposable elements. BMC Genet 2007, 8:78. 
14. Amit M, Sela N, Keren H, Melamed Z, Muler I, Shomron N, Izraeli S, Ast G: Biased exonization of transposed elements in duplicated genes: $A$ lesson from the TIF-IA gene. BMC Mol Biol 2007, 8:109.

15. Krull M, Petrusma M, Makalowski W, Brosius J, Schmitz J: Functional persistence of exonized mammalian-wide interspersed repeat elements (MIRs). Genome Res 2007, 17:1139-1145.

16. Sela N, Mersch B, Gal-Mark N, Lev-Maor G, Hotz-Wagenblatt A, Ast G: Comparative analysis of transposed elements' insertion within human and mouse genomes reveals Alu's unique role in shaping the human transcriptome. Genome Biol 2007, 8:R127.

17. Schwartz SH, Silva J, Burstein D, Pupko T, Eyras E, Ast G: Large-scale comparative analysis of splicing signals and their corresponding splicing factors in eukaryotes. Genome Res 2008, 18:88-103.

18. Alekseyenko AV, Kim N, Lee CJ: Global analysis of exon creation versus loss and the role of alternative splicing in 17 vertebrate genomes. Rna 2007, 13:661-670.

19. Fox-Walsh KL, Dou Y, Lam BJ, Hung SP, Baldi PF, Hertel KJ: The architecture of pre-mRNAs affects mechanisms of splice-site pairing. Proc Natl Acad Sci USA 2005, 102:16176-16181.

20. Roy M, Kim N, Xing Y, Lee C: The effect of intron length on exon creation ratios during the evolution of mammalian genomes. RNA 2008, 14:2261-2273.

21. Talerico M, Berget SM: Intron definition in splicing of small Drosophila introns. Mol Cell Biol 1994, 14:3434-3445

22. Ast G: How did alternative splicing evolve? Nat Rev Genet 2004, 5:773-782.

23. Deininger PL, Moran JV, Batzer MA, Kazazian HH Jr: Mobile elements and mammalian genome evolution. Curr Opin Genet Dev 2003, 13:651-658.

24. Deininger PL, Batzer MA: Alu repeats and human disease. Mol Genet Metab 1999, 67:183-193.

25. Lev-Maor G, Sorek R, Levanon EY, Paz N, Eisenberg E, Ast G: RNA-editingmediated exon evolution. Genome Biol 2007, 8:R29.

26. Sorek R: The birth of new exons: mechanisms and evolutionary consequences. RNA 2007, 13:1603-1608.

27. Gu W, Ray DA, Walker JA, Barnes EW, Gentles AJ, Samollow PB, Jurka J, Batzer MA, Pollock DD: SINEs, evolution and genome structure in the opossum. Gene 2007, 396:46-58.

28. Bejerano G, Lowe CB, Ahituv N, King B, Siepel A, Salama SR, Rubin EM, Kent WJ, Haussler D: A distal enhancer and an ultraconserved exon are derived from a novel retroposon. Nature 2006, 441:87-90.

29. UCSC Genome Browser [http://genome.ucsc.edu]

30. Levy A, Sela N, Ast G: TranspoGene and microTranspoGene: transposed elements influence on the transcriptome of seven vertebrates and invertebrates. Nucleic Acids Res 2008, 36:D47-52

31. Mandal PK, Kazazian HH Jr: SnapShot: Vertebrate transposons. Cell 2008, 135:192-192-e1.

32. Levy A, Schwartz S, Ast G: Large-scale discovery of insertion hotspots and preferential integration sites of human transposed elements. Nucleic Acids Res 38:1515-1530.

33. Krull M, Brosius J, Schmitz J: Alu-SINE exonization: en route to proteincoding function. Mol Biol Evol 2005, 22:1702-1711.

34. Zhang $\mathrm{XH}$, Chasin LA: Comparison of multiple vertebrate genomes reveals the birth and evolution of human exons. Proc Natl Acad Sci USA 2006, 103:13427-13432

35. Kim E, Magen A, Ast G: Different levels of alternative splicing among eukaryotes. Nucleic Acids Res 2007, 35:125-131.

36. Catania F, Lynch M: Where do introns come from? PLoS Bio/ 2008, 6:e283.

37. NCBI Reference Sequence [http://www.ncbi.n/m.nih.gov/refseg/]

38. Sayers EW, Barrett T, Benson DA, Bryant SH, Canese K, Chetvernin V, Church DM, DiCuccio M, Edgar R, Federhen S, Feolo M, Geer LY, Helmberg W, Kapustin Y, Landsman D, Lipman DJ, Madden TL, Maglott DR, Miller V, Mizrachi I, Ostell J, Pruitt KD, Schuler GD, Sequeira E, Sherry ST, Shumway M, Sirotkin K, Souvorov A, Starchenko G, Tatusova TA, et al:: Database resources of the National Center for Biotechnology Information. Nucleic Acids Res 2009, 37:D5-15.

39. Ensembl [http://www.ensembl.org/index.html]

40. Spudich G, Fernandez-Suarez XM, Birney E: Genome browsing with Ensembl: a practical overview. Brief Funct Genomic Proteomic 2007, 6:202-219.

41. Sela N, Stern A, Makalowski W, Pupko T, Ast G: Transduplication resulted in the incorporation of two protein-coding sequences into the Turmoil-1 transposable element of C. elegans. Biol Direct 2008, 3:41.
42. Izsvak Z, Ivics Z, Garcia-Estefania D, Fahrenkrug SC, Hackett PB: DANA elements: a family of composite, tRNA-derived short interspersed DNA elements associated with mutational activities in zebrafish (Danio rerio). Proc Natl Acad Sci USA 1996, 93:1077-1081.

43. Ogiwara I, Miya M, Ohshima K, Okada N: Retropositional parasitism of SINEs on LINEs: identification of SINEs and LINEs in elasmobranchs. Mol Biol Evol 1999, 16:1238-1250

44. Gal-Mark N, Schwartz S, Ram O, Eyras E, Ast G: The pivotal roles of TIA proteins in $5^{\prime}$ splice-site selection of alu exons and across evolution. PLoS Genet 2009, 5:e1000717.

45. Lee JY, Ji Z, Tian B: Phylogenetic analysis of mRNA polyadenylation sites reveals a role of transposable elements in evolution of the 3 '-end of genes. Nucleic Acids Res 2008, 36:5581-5590.

46. Chen LL, DeCerbo JN, Carmichael GG: Alu element-mediated gene silencing. EMBO J 2008, 27:1694-1705.

47. Smalheiser NR, Torvik VI: Mammalian microRNAs derived from genomic repeats. Trends Genet 2005, 21:322-326.

48. Smalheiser NR, Torvik VI: Alu elements within human mRNAs are probable microRNA targets. Trends Genet 2006, 22:532-536.

49. Berget SM: Exon recognition in vertebrate splicing. J Biol Chem 1995 270:2411-2414

50. Ram O, Ast G: SR proteins: a foot on the exon before the transition from intron to exon definition. Trends Genet 2007, 23:5-7.

51. Franchini LF, Ganko EW, McDonald JF: Retrotransposon-gene associations are widespread among D. melanogaster populations. Mol Biol Evol 2004, 21:1323-1331.

52. Gonzalez J, Petrov DA: The adaptive role of transposable elements in the Drosophila genome. Gene 2009, 448:124-133.

53. Aminetzach YT, Macpherson JM, Petrov DA: Pesticide resistance via transposition-mediated adaptive gene truncation in Drosophila. Science 2005, 309:764-767.

54. Kedde M, Agami R: Interplay between microRNAs and RNA-binding proteins determines developmental processes. Cell Cycle 2008, 7:899-903.

55. Kuhn RM, Karolchik D, Zweig AS, Trumbower H, Thomas DJ, Thakkapallayil A, Sugnet CW, Stanke M, Smith KE, Siepel A, Rosenbloom KR, Rhead B, Raney BJ, Pohl A, Pedersen JS, Hsu F, Hinrichs AS, Harte RA, Diekhans M, Clawson H, Bejerano G, Barber GP, Baertsch R, Haussler D, Kent WJ: The UCSC genome browser database: update 2007. Nucleic Acids Res 2007 35:D668-673.

56. Pruitt KD, Tatusova T, Maglott DR: NCBI reference sequences (RefSeq): a curated non-redundant sequence database of genomes, transcripts and proteins. Nucleic Acids Res 2007, 35:D61-65.

57. TranspoGene [http://TranspoGene.tau.ac.il]

58. RepeatMasker [http://www.repeatmasker.org/]

59. Jurka J: Repbase update: a database and an electronic journal of repetitive elements. Trends Genet 2000, 16:418-420.

60. Jurka J, Kapitonov WV, Pavlicek A, Klonowski P, Kohany O, Walichiewicz J: Repbase Update, a database of eukaryotic repetitive elements. Cytogenet Genome Res 2005, 110:462-467

61. Galaxy [http://main.g2.bx.psu.edu/

62. Giardine B, Riemer C, Hardison RC, Burhans R, Elnitski L, Shah P, Zhang Y, Blankenberg D, Albert I, Taylor J, Miller W, Kent WJ, Nekrutenko A: Galaxy: a platform for interactive large-scale genome analysis. Genome Res 2005, 15:1451-1455.

63. The Exon-Intron Database [http://hsc.utoledo.edu/depts/bioinfo/ database.html]

64. Shepelev V, Fedorov A: Advances in the Exon-Intron Database (EID). Brief Bioinform 2006, 7:178-185

65. Dehal P, Satou Y, Campbell RK, Chapman J, Degnan B, De Tomaso A Davidson B, Di Gregorio A, Gelpke M, Goodstein DM, Harafuji N, Hastings KE, Ho I, Hotta K, Huang W, Kawashima T, Lemaire P, Martinez D, Meinertzhagen IA, Necula S, Nonaka M, Putnam N, Rash S, Saiga H, Satake M, Terry A, Yamada L, Wang HG, Awazu S, Azumi K, et al:: The draft genome of Ciona intestinalis: insights into chordate and vertebrate origins. Science 2002, 298:2157-2167.

66. Biemont C, Vieira C: Genetics: junk DNA as an evolutionary force. Nature 2006, 443:521-524.

doi: $10.1186 / \mathrm{gb}-2010-11-6-\mathrm{r} 59$

Cite this article as: Sela et al., The role of transposable elements in the evolution of non-mammalian vertebrates and invertebrates Genome Biology 2010, 11:R59 